\title{
A NEW METHOD OF SEPARATING THE OUTERSEGMENTS OF RODS FROM RETINAL TISSUES
}

\author{
EIICHI KIMURA*

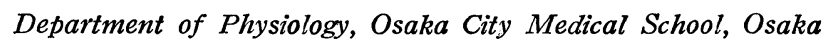

It has been shown by many workers that the outer segments of rods alone contain all the rhodopsin to be found in the retina and the first step of the photoreception of the eye may be enclosed in them $(5,6,8,9,11)$. Recently Wald et al. $(12,13)$ succeeded in the synthesis of rhodopsin from retinene and vitamin A (1950) and described the mechanism of rhodopsin synthesis (4). Collins and Morton (1) obtained unprecedented pure rhodopsin solution with digitonin (1950). According to their detailed experiments, a combination of Saito's (10) sugar floatation method of separating the outersegments of rods from the retina with alum hardening of the rods is superior to the other methods tried, but even by his method some impurities seem to be found in the rhodopsin solution.

The author improved Saito's sugar floatation method in order to separate the outersegments of rods more easily and completely, and extracted rhodopsin from them with distilled water and some inorganic substances. In this paper a new method of separating the outersegments of rods from the retinal tissues is described, which may be used with value to study chemical and morphological changes of rods by light.

\section{METHOD}

Animals are kept in a dark room overnight. All operations are carried out by light from a red dark room lamp. Frogs and toads are decapitated and the eye-balls enuclated from the orbits. With a pair of fine scissors the optic nerve is cut off and a single cut is made right across the equator. Each hind half of the eye-ball is placed in a vessel containig $0.65 \% \mathrm{NaCl}$ solution and the retinae are removed by means of Hosoya's method $(2,3)$. After all the retinae have been removed, several pieces of them are sandwiched at one time between two slide glasses and pressed hard to crush the retinae. All the outersegments of the rods are detached from the retinae by this process (fig. 1).

The crushed retinae are rubbed away into a small vessel containing sugar solution (s.g. 1.200 at $15^{\circ}$ C.). All removed retinae are thus treated. Next, the sugar solution, in which the rods and various fragments of the retinal tissues

Received for publication May 16, 1952.

* 木柇英一 


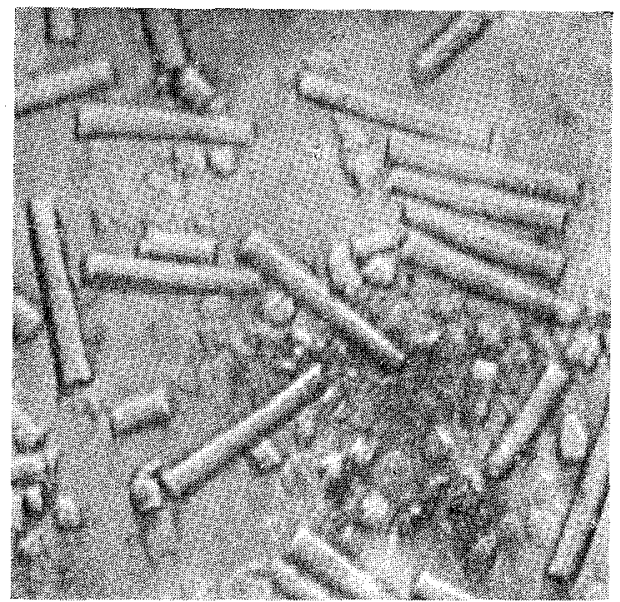

FIG. 1. The crushed retinal tissues and the detached outersegments of rods ( $\times 400$, reduced $3 / 4)$. (Toad)

are suspended is poured into a centrifuge tube. The outersegments of rods can be separated as follows, by the preparation of four layers in the centrifuge tube. The bottom layer consists of sugar solution containing the crushed retinae; the second and third layers contain sugar solutions whose specific gravities are 1.150 and 1.120 respectively. The top layer consists distilled water. The thickness of the three layers from the top must be less than $1 \mathrm{~cm}$. In order to place one layer upon the other, liquids must be poured quietly along the inner wall of the centrifuge tube in turn with a pi-

pette (fig. 2).

Glycerin solutions of various specific gravities are also available for this purpose, their specific gravities being $1.200,1.180$ and 1.150 respectively.

This suspension is centrifuged at about $3000 \mathrm{rpm}$. for $15 \mathrm{~min}$. The outersegments of rods collect at the boundary between the second and third layer and form a thin stratum. This stratum is pipetted off and poured into a centrifuge tube containing sugar solution of s.g. 1.160. To it is added some distilled water to form a layer and centrifuged again at $300 \mathrm{rpm}$. for $15 \mathrm{~min}$. The stratum of outersegments formed at the boundary between the two liquids is poured into a centrifuge tube containing distilled water and centrifuged at $3000 \mathrm{rpm}$. for $10 \mathrm{~min}$. The outersegments sink to the bottom completely, and

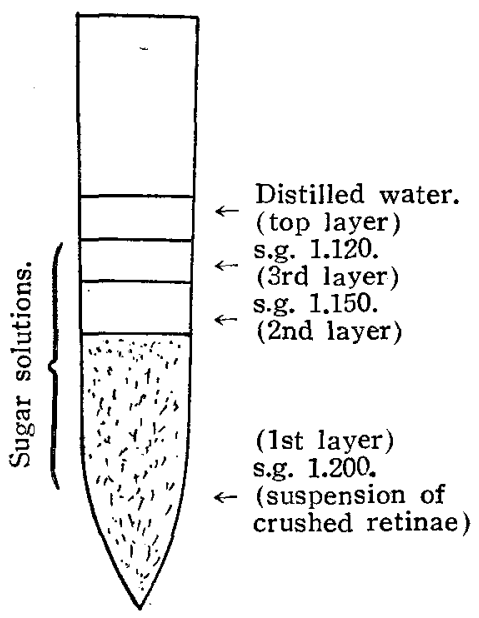

FIG. 2. Four liquid layers in the centrifuge tube. can be obtained in a pure state by decanting the supernatant fluid. Sugar solutions of various specific gravities are prepared from the saturated cane sugar solutions whose $p \mathrm{H}$ should be about 7.0.

\section{RESULTS}

The outersegments of rods collect at the boundary between the second and third layers and form a thin stratum, the pigment epithelium, nuclear and ganglionic layers sink to the bottom, while pigment granules and fine fragments of various cells float at the boundary between the first and the second layer. 


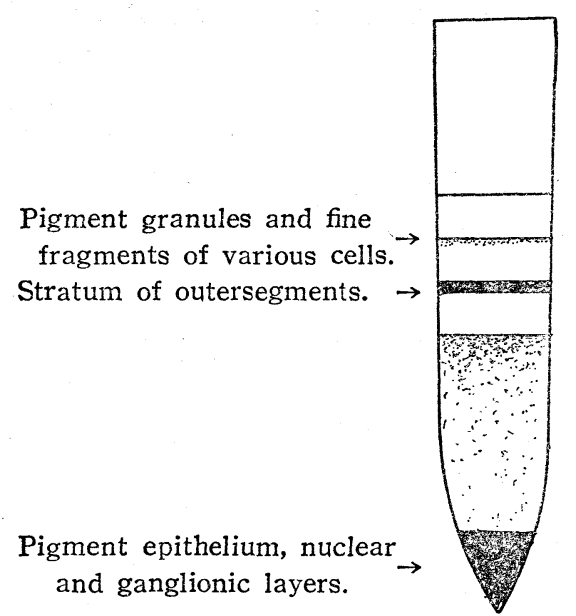

Very light fragments flock together at the boundary between the third and the fourth layers (fig. 3).

The outersegments of rods can be obtained separately by pipetting off their stratum, but microscopically there are a few pigment granules and minute fragments intermingled. By means of the second treatment described above, the impurities can be removed.

Deformation of the outersegments of rods is not marked in spite of immersion in sugar solution of high osmotic pressure.

FIG. 3. Separated retinal tissues in the centrifuge tube.

\section{DISCUSSION}

Lythgoe (7) used a method of shaking vigorously the test tube containing the removed retinae in order to detach the outersegments of rods. This method is inconvenient for mammalian rod outersegments, as they are very fragile and liable to be smashed into pieces.

Saito (10) shook the removed retinae in $45 \%$ sugar solution gently to detach the outersegments of rods. This technique is also difficult in case of mammalian retinae because the rod's outersegments are with difficulty detached. Crushing the retinae with slide glasses is superior to these methods for detaching the outersegments of rods without damage, especially in fishes and mammals. The specific gravity of these detached outersegments is about 1.140. Other cells e.g. pigment epithelium, nuclear and ganglionic layers are heavier (s.g. about 1.240), while some minute fragments and oil drops of pigment epithelium are lighter than the outersegments. The differences in specific gravities make it possible to separate the outersegments of rods from retinal tissues by means of a centrifuge. Saito centrifuged his $45 \%$ sugar solution, in which the outersegments of rods are suspended at $6000 \mathrm{rpm}$. for $15 \mathrm{~min}$. and separated them by floatation. By his method there remain some impuri-

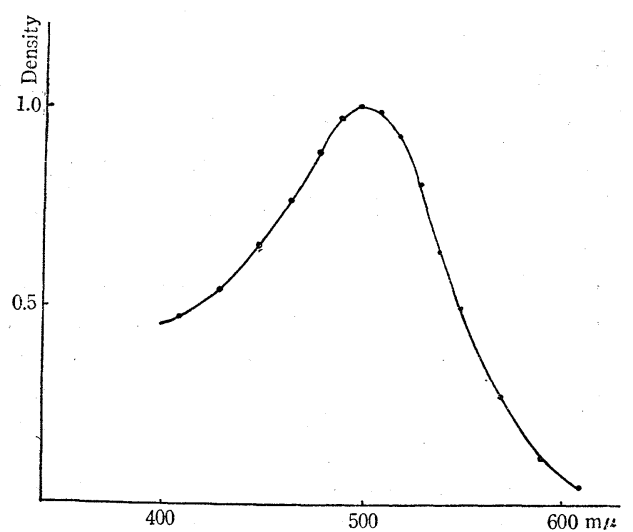

FIG. 4. Absorption curve of rhodopsin extracted with $10 \% \mathrm{NH}_{4} \mathrm{SCN}$ solution. 
ties mixed. In order to remove these impurities, the new method described in this paper may be better. The $p \mathrm{H}$ of the sugar sulutions is of great significance in this method. If the $p \mathrm{H}$ values are below 5.0 good results will not be obtained. Rhodopsin can be extracted from the outersegments of rods with solutions of various substances, where the extraction from whole retinae is not possible (fig. 4).

\section{SUMMARY}

(1) The outersegments of rods are detached from the retinal tissues without damage by crushing the retinae with slides.

(2) The detached outersegments of rods are separated completely by means of a new sugar floatation method.

(3) The process involved in this new sugar floatation method is as follows:

(a) Four liquid layers are prepared in a centrifuge tube. Three of them consist of sugar solutions, of specific gravities $1.200,1.150,1.120$ respectively and the top layer is made up of distilled water. The $p \mathrm{H}$ of them should be 7.0.

(b) Centrifuge at $3000 \mathrm{rpm}$. for $15 \mathrm{~min}$.

(c) The stratum of floating outersegments at the boundary between two solutions is pipetted off and recentrifuged in a sugar solution of s.g. 1.160.

(4) Gylcerin solutions of various specific gravities can be used as substitutes for the sugar solutions; their specific gravities should be $1.200,1.180$ and 1.150 .

(5) Rhodopsin can be extracted from the outersements of rods with various substances hitherto not described.

The author wishes to thank Prof. Yuji Hosoya for his kind advices in this study.

\section{REFERENCES}

1. Collins, F. D. AND Morton, R. A. Biochem. J. $47:$ 3, 1950.

2. Hosoy A, Y. Pfügers Arch. 233 : 57, 1933-4.

3. Hosoya, Y. AND BAYERL, V. Pfiügers Arch. 231 : 563, 1933.

4. HubBard, R. AND Wald, G, Proc. Natl. Acad. Sci. 37: 69, 1951.

5. KüHNE, W. Über den Sehpurpur. Unters. a. d. Physiol. Inst. Heidelberg. 1 : 15, 1877.

6. KüHNE, W. in Hermanns. Handbuch d. Physiol. 3(1) : 235, 1879.

7. Lythgoe, R. J. J. Physiol. 89 : 331, 1937.

8. Morton, R. A. AND GoodWIN, T. W. Nature 153: 405, 1944.

9. NAKAShima, M. Moumaku no Kagaku (Chemistry of the Retina). Tokyo: Sogensha, 1948.

10. SAITO, Z. Tohoku J. exp. Med. $32: 432,1938$.

11. WAld, G. AND HubBard, R. J. gen. Physiol. $32: 367,1948-49$.

12. Wald, G. AND Brown, P. K. Proc. Natl. Acad. Sci. 36(2) : 84, 1950.

13. Wald, G. ANd Hubbard, R. Proc. Natl. Acad. Sci. 36: 92, 1950. 